\title{
Solar-like oscillations in $\beta$ Hydri: Confirmation of a stellar origin for the excess power ${ }^{\star}$
}

\author{
F. Carrier $^{1}$, F. Bouchy ${ }^{1}$, F. Kienzle ${ }^{1}$, T. R. Bedding ${ }^{2}$, H. Kjeldsen ${ }^{3}$, R. P. Butler ${ }^{4}$, I. K. Baldry ${ }^{5}$, \\ S. J. O'Toole ${ }^{2}$, C. G. Tinney ${ }^{5}$, and G. W. Marcy ${ }^{6,7}$ \\ 1 Observatoire de Genève, 1290 Sauverny, Switzerland \\ 2 School of Physics A28, University of Sydney, NSW 2006, Australia \\ 3 Theoretical Astrophysics Center, University of Aarhus, 8000 Aarhus C, Denmark \\ 4 Carnegie Institution of Washington, Department of Terrestrial Magnetism, 5241 Broad Branch Road NW, \\ Washington, DC 20015-1305, USA \\ 5 Anglo-Australian Observatory, PO Box 296, Epping, NSW 1710, Australia \\ 6 Department of Astronomy, University of California, Berkeley, CA 94720, USA \\ 7 Department of Physics and Astronomy, San Francisco, CA 94132, USA
}

Received 14 March 2001 / Accepted 10 August 2001

\begin{abstract}
The G2 subgiant $\beta$ Hyi (HR 98) was observed with the CoRALIE fiber-fed echelle spectrograph on the 1.2-m Swiss telescope at La Silla in June 2000. The resulting 971 high-accuracy radial velocities exhibit an rms scatter of $2.72 \mathrm{~m} \mathrm{~s}^{-1}$ and a mean noise level in the amplitude spectrum of $0.122 \mathrm{~m} \mathrm{~s}^{-1}$. These measurements show significant excess in the power spectrum between $0.7-1.4 \mathrm{mHz}$ with $0.40 \mathrm{~m} \mathrm{~s}^{-1}$ peak amplitude. Fitting the asymptotic relation to the power spectrum gives a large splitting of $58 \mu \mathrm{Hz}$ which is in good agreement with theoretical expectations. Our data confirm the independent detection of solar-like oscillations in $\beta$ Hyi announced by Bedding et al. (2001) which used the UCLES echelle spectrograph on the 3.9-m Anglo-Australian Telescope. Two independent instruments and calibration methods obtained very similar power excesses thus leaving little doubts on the stellar origin of the detected signal.
\end{abstract}

Key words. stars: oscillations - stars: individual: $\beta$ Hydri - techniques: radial velocities

\section{Introduction}

The success of helioseismology encourages corresponding investigations on other stars. The measurement of frequencies and amplitudes of $p$-mode oscillations for solarlike stars provides an insight into the internal structure and is nowadays the most powerful constraint to the theory of stellar evolution (see Guenther \& Demarque 1993). Theoretical amplitude predictions for solar-like stars have been proposed by Christensen-Dalsgaard \& Frandsen (1983) and Houdek et al. (1999). Until 1999, various techniques, such as photometry, radial velocity or equivalent width measurements, gave either only upper

Send offprint requests to: F. Carrier, e-mail: Fabien.Carrier@obs.unige.ch

* Based on observations collected with the CorALIE echelle spectrograph on the 1.2-m Euler Swiss telescope at La Silla Observatory, ESO Chile. limits on the $p$-mode oscillations or not very convincing as well as non-reproducible detections. Until now, only a few bright stars have been studied (see reviews given by Kjeldsen \& Bedding 1995a; Heasley et al. 1996; Bedding \& Kjeldsen 1998). Very good recent results were obtained by Martic et al. (1999) on Procyon with the Elodie spectrograph on the 1.93-m telescope of Observatoire de HauteProvence (France).

A good candidate to detect $p$-mode oscillations is the bright southern star $\beta$ Hyi (HR 98, $m_{v}=2.80$, G2 IV) with a luminosity of $3.5 L_{\odot}$, a mass of $1.1 M_{\odot}$ and $6.7 \mathrm{~Gy}$ old (Dravins et al. 1998). Edmonds \& Cram (1995) attempted to detect oscillations on this star with radial velocity measurements, from which they obtained an upper limit of $1.5-2 . \mathrm{m} \mathrm{s}^{-1}$ for the amplitudes of the strongest modes.

To tackle the detection of $\beta$ Hyi oscillations, with an expected amplitude of $0.64 \mathrm{~m} \mathrm{~s}^{-1}$ centred at $1.1 \mathrm{mHz}$ with 


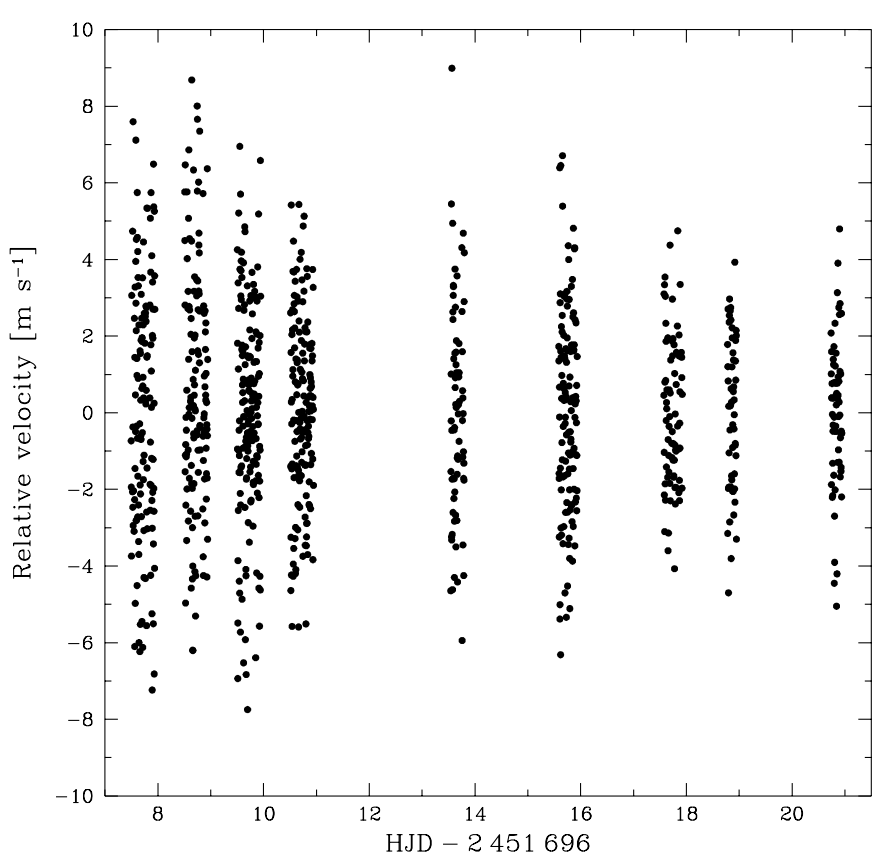

Fig. 1. Radial velocities of $\beta$ Hyi relative to a reference spectrum taken during each night (the best spectrum of the night). The dispersion reaches $2.72 \mathrm{~m} \mathrm{~s}^{-1}$.

a primary frequency splitting $\Delta \nu_{0}=64 \mu \mathrm{Hz}$ (Kjeldsen \& Bedding 1995a), a two-site campaign involving CORALIE on the 1.2-m Swiss Telescope (La Silla, Chile) and UCLES on the 3.9-m Anglo-Australian Telescope (Siding Spring Observatory, Australia) was organised. The detection using UCLES of excess power, centred at $1.0 \mathrm{mHz}$ with a peak amplitude of $0.5 \mathrm{~m} \mathrm{~s}^{-1}$ was recently reported in Bedding et al. (2001). In the following sections, the independent detection resulting from CORALIE data analysis is presented.

\section{Observations and data reduction}

$\beta$ Hyi was observed over a campaign of two weeks (720 June 2000) with CORALIE, the high-resolution fiberfed echelle spectrograph (Queloz et al. 2000) mounted on the 1.2-m Swiss telescope at La Silla (ESO, Chile). CorAliE, known for its exo-planet program, is an improved version of the ELODIE spectrograph (Baranne et al. 1996). Thanks to a slightly different optical combination at the entrance of the spectrograph and the use of a $2 \mathrm{k}$ by $2 \mathrm{k}$ CCD camera with smaller pixels $(15 \mu \mathrm{m})$, CoRAliE reaches a resolving power of $50000(\lambda / \Delta \lambda)$ with a 3 pixels sampling. During stellar exposures, a separated reference fiber is continuously illuminated by a thoriumargon lamp, in order to monitor the spectrograph stability and thus to obtain high-accuracy velocity measurements. Spectra from CORALIE were extracted at the telescope, using INTER-TACOS (INTERpreter for the Treatment, the Analysis and the COrrelation of Spectra) software package developed by D. Queloz and L. Weber at the Geneva Observatory (Baranne et al. 1996). The wavelength coverage of these spectra is $3875-6820 \AA$, recorded on 68 orders.
Table 1. Distribution and dispersion of data obtained with Coralie.

\begin{tabular}{cll}
\hline Date & Nb spectra & $\sigma\left[\mathrm{m} \mathrm{s}^{-1}\right]$ \\
\hline $2000 / 06 / 07$ & 134 & 3.34 \\
$2000 / 06 / 08$ & 130 & 3.18 \\
$2000 / 06 / 09$ & 162 & 2.81 \\
$2000 / 06 / 10$ & 155 & 2.40 \\
$2000 / 06 / 11$ & 38 & - \\
$2000 / 06 / 12$ & 13 & - \\
$2000 / 06 / 13$ & 73 & 2.81 \\
$2000 / 06 / 14$ & - & - \\
$2000 / 06 / 15$ & 124 & 2.66 \\
$2000 / 06 / 16$ & - & - \\
$2000 / 06 / 17$ & 76 & 1.94 \\
$2000 / 06 / 18$ & 54 & 2.02 \\
$2000 / 06 / 19$ & - & - \\
$2000 / 06 / 20$ & 63 & 1.94 \\
\hline
\end{tabular}

In order to reach a signal-to-noise ratio close to 100 , 120 -s exposures with 108-s dead-times in-between were taken. The standard radial velocity computation by a cross-correlation algorithm was replaced by an algorithm based on the optimum weight procedure (Connes 1985; Bouchy et al. 2001). A small drift, less than $5 \mathrm{~m} \mathrm{~s}^{-1}$, appears at the beginning of each night due to the atmospheric dispersion corrector, which does not work above an airmass of 2 , while the star began to be observed with an airmass of about 3.5. However, this effect can be entirely eliminated by a low order polynomial fit subtraction without consequences for the characterization of high frequency oscillation modes.

A total of 14 nights were allocated in June 2000 for the observation of $\beta$ Hyi. The poor weather conditions prevented us from observing during 3 whole nights and the other 11 nights were not all complete. The distribution and the dispersion of the radial velocities are listed in Table 1. The nights of June 11 and 12 were cloudy and have not enough measurements to be include in the analysis. Some bad points, suspected to be introduced by guiding and atmospheric dispersion corrector errors, were removed. The signal-to-noise ratio, in the range 40 to 110 , depends on the seeing, the extinction and the airmass. The 971 radial velocities are shown in Fig. 1 . The rms scatter of these measurements is $2.72 \mathrm{~ms}^{-1}$. The uncertainties due to photon and detector noise vary between 1.1 and $4 \mathrm{~ms}^{-1}$.

\section{Stellar power spectra analysis}

In order to compute the power spectrum of the velocity time series, we use the Lomb-Scargle modified algorithm (Lomb 1976; Scargle 1982) with a weight being assigned to each point according its uncertainty estimate. The time scale gives a resolution of $0.86 \mu \mathrm{Hz}$. Figure 2 


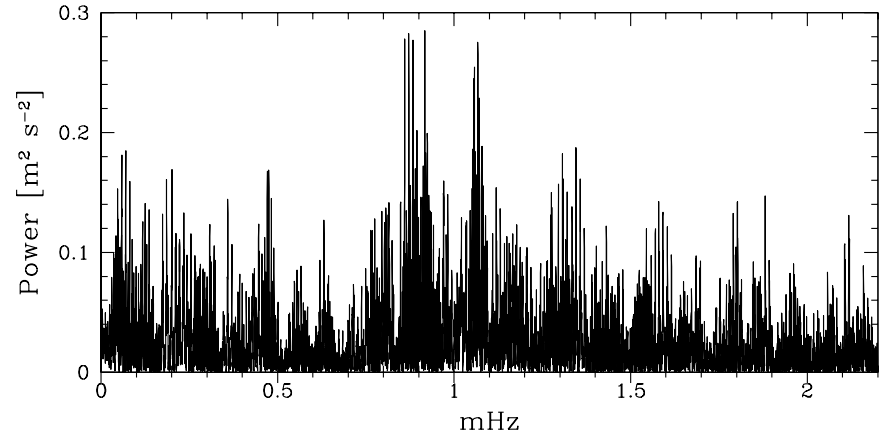

Fig. 2. Power spectrum of Doppler shift measurements for $\beta$ Hyi.

shows this periodogram. The mean white noise level $\sigma_{\text {pow }}$ above $1.6 \mathrm{mHz}$ is $0.019 \mathrm{~m}^{2} \mathrm{~s}^{-2}$. With 971 measurements, the velocity accuracy corresponds thus to $2.15 \mathrm{~ms}^{-1}$.

The power spectrum presents an excess in the range $0.7-1.4 \mathrm{mHz}$ with several significant peaks near $1 \mathrm{mHz}$ degraded by the complicated window function. The amplitude of the strongest peaks is computed according to the formulae of Kjeldsen \& Bedding (1995a) which take into account the noise effect:

$\left(A_{\mathrm{osc}}\right)^{2}=\left(A_{1}\right)^{2}-(8.7 \pm 2.3) \sigma_{\mathrm{amp}}^{2}$

$\sigma_{\mathrm{amp}}=\sqrt{\pi \sigma_{\mathrm{pow}} / 4}$

$A_{1}$ is the amplitude of the strongest peak, $\sigma_{\text {amp }}$ and $\sigma_{\text {pow }}$ are the mean noise levels in the amplitude and in the power spectrum respectively, and $A_{\mathrm{osc}}$ is the searched "true" oscillation amplitude. We obtain $\sigma_{\mathrm{amp}}=0.122 \mathrm{~m} \mathrm{~s}^{-1}$ and $A_{\mathrm{osc}}=0.40 \pm 0.05 \mathrm{~m} \mathrm{~s}^{-1}$.

Considering that the window function of our whole run is complicated, due to bad weather, only the three best consecutive nights (June 8-10) were selected to determine the large splitting $\Delta \nu$ (see Fig. 3). The time scale of these 3 nights gives a resolution of $4.75 \mu \mathrm{Hz}$. The mean white noise level $\sigma_{\text {pow }}$, computed above $1.6 \mathrm{mHz}$, reaches $0.034 \mathrm{~m}^{2} \mathrm{~s}^{-2}$, which corresponds, with 447 measurements, to a velocity accuracy of $1.95 \mathrm{~m} \mathrm{~s}^{-1}$. The amplitude of the strongest mode, which appears at the same frequency than in the power spectrum of Fig. 2, corresponds to $0.58 \pm 0.06 \mathrm{~m} \mathrm{~s}^{-1}$. According to the asymptotic relation, a comb response of the power spectrum is calculated (Kjeldsen et al. 1995b). The average comb response (see Fig. 3) is computed using all peaks greater than $0.22 \mathrm{~m}^{2} \mathrm{~s}^{-2}$ (i.e. with $S / N$ greater than 2.5 in the amplitude spectrum) in the frequency range $0.6-1.5 \mathrm{mHz}$. The large splitting $\Delta \nu$ has a value of $58 \mu \mathrm{Hz}$. This parameter is in agreement with the expected value of $64 \pm 5 \mu \mathrm{Hz}$ scaling from the Sun.

\section{Comparison with UCLES results}

The amount of excess power in the UCLES and CoRALIE power spectra are both centred near $1 \mathrm{mHz}$ and cover the same frequency range $0.7-1.4 \mathrm{mHz}$.
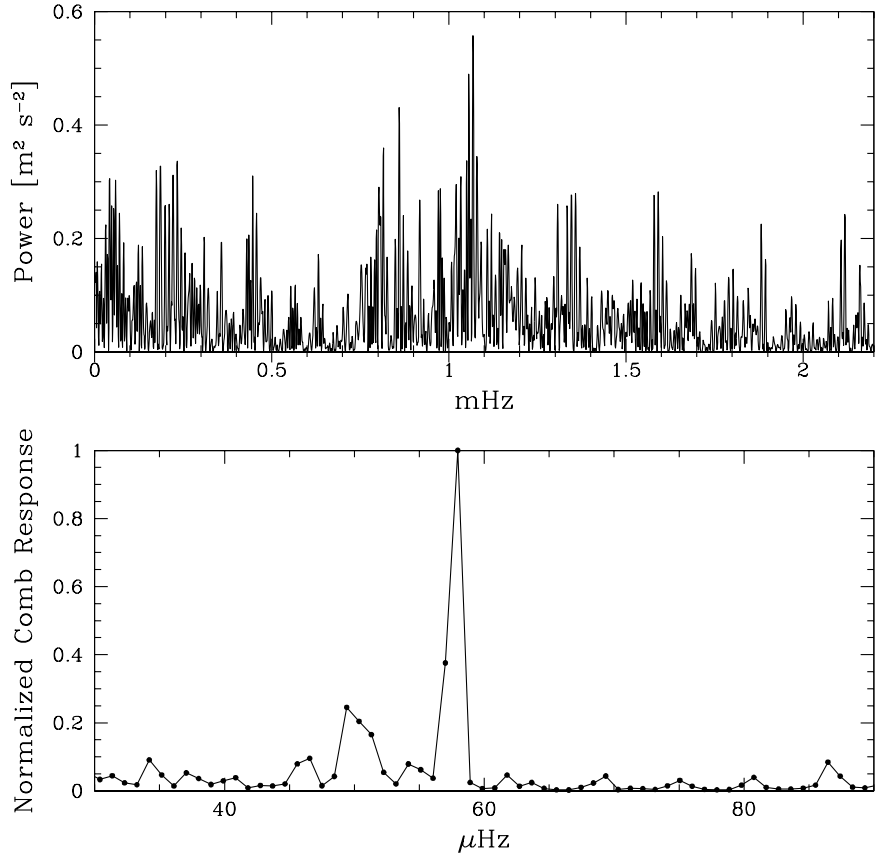

Fig. 3. Top: power spectrum of the radial velocities of $\beta$ Hyi for the three best consecutive nights. The time scale for these nights gives a resolution of $4.75 \mu \mathrm{Hz}$. Bottom: average Comb Response computed with $0.95 \mu \mathrm{Hz}$ resolution.

The strongest oscillation amplitude reaches $0.4 \mathrm{~m} \mathrm{~s}^{-1}$ for our whole run and $0.58 \mathrm{~m} \mathrm{~s}^{-1}$ for our three best consecutive nights. These values are in good agreement with the value of $0.5 \mathrm{~m} \mathrm{~s}^{-1}$ found by Bedding et al. (2001). Indeed, the oscillation modes have finite lifetimes, because they are being continually damped. Thus, if we observed the star during a longer time series, the signal is weakened due to this finite lifetimes. Bedding et al. observed $\beta$ Hyi during five nights and found an amplitude contained between the values deduced from our three best consecutive nights and from our whole run of fourteen nights. This suggests that the mode lifetimes in $\beta$ Hyi may be several days.

The large splitting $\Delta \nu=58 \mu \mathrm{Hz}$, deduced from the $4.75 \mu \mathrm{Hz}$ resolution power spectrum of our three best consecutive nights, confirms the results from Bedding et al. who found two possible solutions: 56.2 and $60.3 \mu \mathrm{Hz}$.

A full analysis of the combined data is beyond the scope of this paper. However, we have checked the effect of deliberately shifting the relative time stamps of the two series. The amplitudes of the strongest peaks in the combined power spectrum are maximized when this shift is zero and become significantly reduced when the offset exceeds one minute. This gives strong evidence that highest peaks in the combined power spectrum have a stellar origin. It also gives gratifying confirmation that the time stamps at both observatories have been correctly calibrated.

All these points confirm that both systems were measuring the same power excess. 


\section{Conclusion}

The G2 subgiant $\beta$ Hyi was observed over 14 nights with the Coralie spectrograph at the Swiss 1.2-m telescope. Although poor weather conditions complicated the spectral window function and thus the analysis of the power spectrum, a significant excess appears in the power spectrum between $0.7-1.4 \mathrm{mHz}$, centred around $1 \mathrm{mHz}$, with a peak amplitude of $0.40 \mathrm{~m} \mathrm{~s}^{-1}$. The frequency spacing is $58 \mu \mathrm{Hz}$. These values are in agreement with theoretical expectations.

This excess of power confirms the results obtained by Bedding et al. (2001). It was detected independently with two instruments (CORALIE and UCLES) on two different sites and using two different radial velocity methods (simultaneous thorium and iodine absorption cell). These two independent detections point out the evidence of solar-like oscillations on $\beta$ Hyi. These two spectrographs show that improvements in radial velocity measurements lead to significant progress in asteroseismology programs.

Acknowledgements. The authors warmly thank F. Pepe and D. Queloz for given advises and encouragements during the data reduction. M. Mayor is greatly acknowledged for his generous time allocation. Also, we thank Conny Aerts for agreeing to swap observing time on CORALIE to allow our observations to be simultaneous with the AAT observations. This work was supported financially by the Swiss National Science Foundation (F. C., F. B. and F. K.), the Australian Research Council (T. R. B. and S. J. O'T.), National Science Foundation Grant AST-9988087 (R. P. B.), SUN Microsystems, and the
Danish Natural Science Research Council and the Danish National Research Foundation through its establishment of the Theoretical Astrophysics Center (H. K.).

\section{References}

Baranne, A., Queloz, D., Mayor, M., et al. 1996, A\&AS, 119, 1 Bedding, T. R., \& Kjeldsen, H. 1998, in Tenth Cambridge Workshop on Cool Stars, Stellar systems and the Sun, ed. Donahue \& Bookbinder, ASP. Conf. Ser., 154, 301

Bedding, T. R., Butler, R. P., Kjeldsen, H., et al. 2001, ApJ, 549, L105

Bouchy, F., Pepe, F., \& Queloz, D. 2001, A\&A, submitted

Christensen-Dalsgaard, J., \& Frandsen, S. 1983, Sol. Phys., 82, 469

Connes, P. 1985, Ap\&SS, 110, 211

Dravins, D., Lindegren, L., \& van den Berg, D. A. 1998, A\&A, 330, 1077

Edmonds, P. D., \& Cram, L. E. 1995, MNRAS, 276, 1295

Guenther, D. B., \& Demarque, P. 1993, ApJ, 405, 298

Heasley, J. N., Janes, K., Labonte, B., et al. 1996, PASP, 108, 385

Houdek, G., Balmforth, N. J., Christensen-Dalsgaard, J., \& Gough, D. O. 1999, A\&A, 351, 582

Kjeldsen, H., \& Bedding, T. R. 1995a, A\&A, 293, 87

Kjeldsen, H., Bedding, T. R., Viskum, M., \& Frandsen, S. 1995b, AJ, 109, 1313

Lomb, N. R. 1976, Astrophys. Space Sci., 39, 447

Martic, M., Schmitt, J., Lebrun, J.-C., et al. 1999, A\&A, 351, 993

Queloz, D., Mayor, M., Weber, L., et al. 2000, A\&A, 354, 99

Scargle, J. D. 1982, ApJ, 263, 835 